\title{
Helicons and magnetoimpurity waves in layered conductors
}

\author{
V. M. Gvozdikov, A. M. Ermolaev, and R. Vega-Monroy \\ Department of Physics, Kharkov State University, 310077 Kharkov, Ukraine \\ E-mail: vladimir.m.gvozdikov@univer.kharkov.ua \\ Received January 28, 1999
}

\begin{abstract}
It is shown that local electron states, caused by impurities in a layered conductor placed in an external magnetic field, give rise to resonant corrections $\delta \sigma_{\alpha \beta}(\omega)$ to the high-frequency conductivity tensor $\delta \sigma_{\alpha \beta}(\omega)$ of the layers. These corrections appear due to the resonant transitions of electrons between the Landau levels and the local states and change dramatically the spectrum of collective electromagnetic oscillations in the system because of the «branch crossing» nearby the frequency $\omega_{0}\left(\hbar \omega_{0}\right.$ is the local state energy). As a result, a new magnetoimpurity wave, $\omega_{-}(k)$, appears in the spectrum in addition to the helicon mode, $\omega_{+}(k)$, which is known to exist in a pure layered conductor in a perpendicular magnetic field ( $k$ is the wave vector along the magnetic field). In the long wavelength limit, $k a<<1$, the helicon-like mode $\omega_{+}(k)$ has a gap of the order of $\omega_{0}$, whereas the magnetoimpurity mode in this limit goes to zero $\omega_{-}(k) \sim(k a)^{2}$ ( $a$ is the distance between adjacent layers). The small damping of these modes due to the broadening of the Landau levels and the magnetoimpurity levels are also calculated.
\end{abstract}

PACS: 73.20.Dx, 72.20.My, 73.20.Mf

\section{Introduction}

Impurities in metals play a twofold role. First, they decrease the free path of electrons and thereby damp electromagnetic waves [1,2]. On the other hand, impurities may create bound states which result in a dramatic change in the electron energy spectrum and create new electromagnetic modes in conventional metals.

The effect of impurities on the electronic energy spectrum becomes much more pronounced in an external magnetic field which effectively decreases the dimensionality of the system and assists the localization of electrons by attractive impurities. Local and quasi-local magnetoimpurity states (i.e., the ones localized nearby the impurity in an external magnetic field) were studied long ago in conventional metals $[3,4]$ and recently in two-dimensional (2D) conductors [5].

Impurities break down the spatial homogeneity of a system, they lift up the degeneracy of the electronic energy spectrum on the Landau orbit center position and split off one local state from each Landau level. The magnitude of splitting, $\Delta$, is equal to the energy of a bound state. Thus, a new resonant frequency $\omega_{s}=\Delta+s \Omega(\Omega=e H / m c$ is the cyclotron frequency and the integer $s=0,1, \ldots$ numerates the resonances) related to electron transitions between the Landau levels and the magnetoimpurity ones appears in the conductivity tensor $\sigma_{\alpha \beta}(\omega)$ and gives rise to the resonant attenuation of the electromagnetic waves in metals [5].

The conductivity tensor, as is well known, is the key quantity in the theory of electromagnetic waves in metals; it determines the dispersion of the electromagnetic waves in conventional [1,2] and layered metals [6-13] and superconductors [14].

The high-frequency conductivity tensor of a $2 D$ electron gas with magnetoimpurity states in quantizing perpendicular magnetic field was calculated in [5] and we are going to apply in what follows the results of these calculations to studies of magnetoimpurity electromagnetic waves in layered conductors.

Electromagnetic waves in layered conductors and superlattices in a quantizing external magnetic field have been studied in a number of works [6-13]. It was found that a quasi-two-dimensional nature of layered conductors brings some specific features compared to the results obtained for conventional $3 D$ metals. In particular, a new type of spiral waves in layered conductors under the conditions of the quantum Hall effect have been predicted theoretically $[9,11-13]$. In these works a model for layered 
electron gas was employed which ignores electron hopping across the layers because the quantum Hall effect takes place in purely $2 D$ systems. Thus, the most interesting case of quasi-two-dimensional behavior of electrons in superlattices corresponds to the model without electron dispersion across the layers. In this model electromagnetic waves can propagate only owing to the interlayer electromagnetic correlations described by the Maxwell's equations. The relationship between the electromagnetic field and the current within the layer is determined by the conductivity tensor which depends on the layer structure and determines the shape of the electromagnetic wave dispersion in layered conductors.

The purpose of the present paper is to calculate the dispersion relation for a new weakly damped electromagnetic wave caused by the resonant transitions between magnetoimpurity levels and the Landau levels in layered conductors in perpendicular to layers quantizing magnetic field.

In Sec. 2 we discuss the physics of the high-frequency conductivity in a $2 D$ conductor with magnetoimpurity states. Section 3 is devoted to calculations of the dispersion relations for helicons and magnetoimpurity electromagnetic waves in layered conductors. Summary of the results obtained and the discussion are given in Sec. 4.

\section{The conductivity tensor in a $2 D$ conductor with magnetoimpurity states}

The helicons in layered conductors at low temperatures have a dispersion along the magnetic field that is described by the function [9]

$$
\omega(k)=\Omega W(k a) .
$$

It turned out that their damping [15] depends on the wave vector $k$ by dint of the same function $W(k a)$ (see Sec. 3 for details):

$$
\gamma(k)=v W(k a),
$$

where

$$
W(k a)=\frac{\sin ^{2}(k a / 2)}{\left(\omega_{*} / 2\right)^{2}+\sin ^{2}(k a / 2)} .
$$

Here $v$ is the electronic collision frequency due to impurities and lattice defects; $\omega_{*}=\omega_{p} a / c, a$ stands for the interlayer spacing; $c$ is the velocity of light and $\omega_{p}$ is the plasma frequency. We have assumed above that $v$ is a smooth function of the energy near the Fermi level. This holds true, in particular, for potential scattering of electrons by impurities.
Generally, the role of impurities is more complicated, because of the possibility of creating bound states. This possibility is enhanced in an external magnetic field and in low-dimensional systems. In the $2 D$ system, an isotropic potential-well of arbitrary small intensity produces a bound state with the energy [16]

$$
\varepsilon_{l}=-\frac{\hbar^{2}}{2 m b^{2}} \exp \left(-\frac{\hbar^{2}}{2 m b^{2} U_{0}}\right) .
$$

Here $b$ is the radius and $U_{0}$ is the depth of the potential well; $m$ stands for the electron mass.

The external magnetic field applied perpendicular to a $2 D$ conductor with impurities produces a host of local states. Impurities lift up the degeneracy on the Landau orbit center position and split off one level from each of the Landau levels. Depending on the sign of the potential the local impurity level may appear either above or below Landau level (correspondingly, for repulsive and attractive potentials). Therefore, the energy spectrum of considered systems consists of a series of two sets of levels: the impurity levels and the Landau ones. The separation between the impurity level and the nearest Landau level in case of a weak attractive potential $U_{0}<\kappa \Omega$ is equal to

$$
\Delta=2 U_{0}\left(\frac{b}{l}\right)^{2},
$$

here $l$ denotes the magnetic length $l=(\hbar c / e H)^{1 / 2}$.

The local energy levels $\varepsilon_{k}^{l}$ correspond to poles of the scattering amplitude of electrons on the complex energy plane and cannot be treated perturbatively. Therefore, it is necessary to take into account exact values of the scattering amplitude $\Psi(\varepsilon)$ for calculations of the conductivity tensor of a $2 D$ conductor in the quantizing magnetic field.

Near the pole it becomes equal to

$$
\Psi(\varepsilon) \approx \frac{R_{k}}{\varepsilon-\varepsilon_{k}^{l}} .
$$

$R_{k}$ in Eq. (6) is the residue of the scattering amplitude $\Psi(\varepsilon)$ at the energy $\varepsilon=\varepsilon_{k}^{l}$.

If $\Delta<<\hbar \Omega$, then

$$
R_{k}=2 \pi(l \Delta)^{2} .
$$

This quantity determines corrections to the conductivity tensor $\sigma_{\alpha \beta}(\omega, H)$ of a $2 D$ electron gas in quantizing perpendicular magnetic field due to the localization of electrons nearby the impurity atoms. Such corrections in linear approximation on the impurities concentration $n_{i}$ have been calculated in 
the paper [5]. The corresponding results for the diagonal $\delta \sigma_{x x}$ and the Hall $\delta \sigma_{x y}$ components of the conductivity tensor are

$$
\begin{aligned}
\delta \sigma_{x x}= & i \frac{e^{2} n_{e}}{m \omega}\left[\sum_{s=0}^{\infty} \alpha_{x x}^{(s)} \frac{\omega_{s}}{\omega-\omega_{s}+i v}+\right. \\
& \left.+\sum_{p=1}^{\infty} \beta_{x x}^{(p)} \frac{\omega_{p}}{\omega-\omega_{p}+i v}\right], \\
\delta \sigma_{x y}= & \frac{e^{2} n_{e}}{m \omega}\left[\sum_{s=0}^{\infty} \alpha_{x y}^{(s)} \frac{\omega_{s}}{\omega-\omega_{s}+i v}+\right. \\
& \left.+\sum_{p=1}^{\infty} \beta_{x y}^{(p)} \frac{\omega_{p}}{\omega-\omega_{p}+i v}\right] .
\end{aligned}
$$

Here $\omega_{s}$ and $\omega_{p}$ are the frequencies of electron transitions between the Landau and the local levels, $\Gamma=\hbar v$ stands for their broadening and determines thereby the quantity $v$. Symbols $\alpha_{i k}^{(s)}$ and $\beta_{i k}^{(p)}$ denote the oscillator strength for the resonant transitions. These quantities depend on the wave vector $q$ by dint of terms of the order $n_{i}(q l)^{2}$ which may be discarded in the limit $n_{i}(q l)^{2}<<1$ ( $q$ is the absolute value of the in-plane wave-vector).

Under above conditions, we have

$$
\begin{gathered}
\alpha_{x x}^{(s)}=\alpha_{0} \sum_{k} R_{k}\left[f\left(\varepsilon_{k}^{l}\right)-f\left(\varepsilon_{k+s}\right)\right] P_{k s}^{+}, \\
\alpha_{x y}^{(s)}=\alpha_{0} \sum_{k} R_{k}\left[f\left(\varepsilon_{k}^{l}\right)-f\left(\varepsilon_{k+s}\right)\right] P_{k s}^{-}, \\
P_{k s}^{ \pm}=\left[\frac{k+s}{\left(\omega_{s}-\Omega\right)^{2}} \pm \frac{k+s+1}{\left(\omega_{s}+\Omega\right)^{2}}\right], \\
\alpha_{0}=\frac{n_{i}}{2 \pi \hbar m l^{4} \omega_{s} n_{e}} .
\end{gathered}
$$

Here $\varepsilon_{n}=\hbar \Omega(n+1 / 2)$ and $f(\varepsilon)$ is the Fermi function.

We do not use any specific form of the impurity potential in Eqs. (10)-(13) so that the quantities $\varepsilon_{k}^{l}, \Gamma, R_{k}$ may be considered as fitting parameters of the theory or may be calculated for some particular scattering potential.

In all above equations, frequencies $\omega_{s}=\omega_{0}+s \Omega$ correspond to transitions from the local levels to Landau levels, whereas frequencies $\omega_{p}=p \Omega-\omega_{0}$ correspond to transitions in the opposite direction $\left(\omega_{0}=\Delta / \hbar\right)$. The summation in Eqs. (10) and (11) is only taken over those local levels which are involved into transitions at given frequency. Near the resonant frequency $\omega \approx \omega_{0}$ only resonant terms with $s=0$ are left, so that

$$
\begin{gathered}
\delta \sigma_{x x} \approx i \frac{e^{2} n_{e}}{m \omega} \alpha_{x x}^{(0)} \frac{\omega_{0}}{\omega-\omega_{0}+i \nu}, \\
\delta \sigma_{x y} \approx \frac{e^{2} n_{e}}{m \omega} \alpha_{x y}^{(0)} \frac{\omega_{0}}{\omega-\omega_{0}+i \nu} .
\end{gathered}
$$

The number of the terms in the sum over $k$ in Eqs. (10), (11) depends on the chemical potential value $\mu$. For example, in case of an attractive impurity potential and also under the condition $\varepsilon_{N}^{l}<\mu<\varepsilon_{N}$ only one term with $k=N$ contributes to the sums (10) and (11). Thus, taking into account Eq. (7) and the inequality $\omega_{0}<<\Omega$ we have, from Eqs. (10) and (11),

$$
\begin{gathered}
\alpha_{x x}^{(0)}=-\frac{n_{i}}{n_{e}} \frac{\omega_{0}}{\Omega}(2 N+1), \\
\alpha_{x y}^{(0)}=\frac{n_{i}}{n_{e}} \frac{\omega_{0}}{\Omega} .
\end{gathered}
$$

$N$ is the number of filled Landau levels, $N=[\mu / \hbar \Omega]$. The symbol $[X]$ here denotes the integer part of $X$.

\section{Helicons and magnetoimpurity waves}

Having at hand the conductivity tensor of a $2 D$ electron gas in quantizing magnetic field [17]

$$
\begin{array}{cc}
\sigma_{x x}=\sigma_{0}\left(1+\gamma^{2}\right)^{-1}, & \sigma_{x y}=-\sigma_{0}+\gamma \sigma_{x x} \\
\sigma_{x x}=\sigma_{y y}, & \sigma_{x y}=-\sigma_{y x}
\end{array}
$$

where

$$
\sigma_{0}(H)=\frac{n_{e} e^{2}}{m \Omega}, \quad \gamma=(v-i \omega) / \Omega
$$

and corrections to $\sigma_{\alpha \beta}$ due to the magnetoimpurity bound states (14)-(17), we now turn to the problem of the electromagnetic wave propagation in layered conductors in an external magnetic field. The dispersion equation for electromagnetic waves in a uniform layered conductor in a perpendicular to conducting layers external magnetic field $H$ was obtained in a number of works $[9,10,15]$. According to [15], it says

$$
\operatorname{det}\left[\delta_{\alpha \beta}-\frac{2 \pi i \omega}{c^{2} q_{\omega}} \sigma_{\alpha \beta}(q, \omega, H) V_{\alpha \beta} S(q, k, \omega)\right]=0 \text {. }
$$


Here $\sigma_{\alpha \beta}$ is the $2 D$ conductivity tensor of a layer (the model employed neglects electron hopping between the layers, so that they are correlated only via the electromagnetic fields); $S(q, k, \omega)$ stands for the structural factor:

$$
\begin{gathered}
S(q, k, \omega)=\frac{\sinh q_{\omega} a}{\cosh q_{\omega} a-\cos k a}, \\
q_{\omega}^{2}=q^{2}-\frac{\omega^{2} \varepsilon_{0}}{c^{2}}, \\
V_{11}=V_{12}=1, V_{22}=V_{21}=-\frac{c^{2} q_{\omega}^{2}}{\omega^{2} \varepsilon_{0}},
\end{gathered}
$$

$q$ is the in-plane wave vector; $k$ is the wave vector component perpendicular to the layers (i.e., parallel to the field $H) ; \varepsilon_{0}$ is the dielectric constant of a substance between the layers.

The dispersion equation (21) is rather general since it determines the frequency $\omega=\omega(q, k, H)$ of a wave which can propagate in the volume of a layered conductor as a function of the wave vector $q, k$ and the field $H$ provided that the conductivity tensor of the constituent layers is known.

Substituting Eqs. (18)-(20) and (14), (15) in Eq. (21), we have

$$
\begin{gathered}
1+\gamma^{2}+i \gamma S \sigma_{0}(\beta-\alpha)+\alpha \beta\left(S \sigma_{0}\right)^{2}=V\left(\alpha_{\|}, \alpha_{\perp}\right) \\
V\left(\alpha_{\|}, \alpha_{\perp}\right)=-\alpha \beta\left(S \sigma_{0}\right)^{2}\left[2 \frac{\gamma \alpha_{\|}-i \alpha_{\perp}}{\gamma+\gamma_{0}}+\right. \\
\left.+\frac{1+\gamma^{2}}{\left(\gamma+\gamma_{0}\right)^{2}}\left(\alpha_{\|}^{2}-\alpha_{\perp}^{2}\right)\right]-S \sigma_{0}(\beta-\alpha) \frac{i \alpha_{\|}\left(1+\gamma^{2}\right)}{\gamma+\gamma_{0}} .
\end{gathered}
$$

We have introduced here the following notations:

$$
\begin{gathered}
\alpha=\frac{2 \pi \omega}{q_{\omega} c^{2}}, \beta=\frac{2 \pi q_{\omega}}{\omega \varepsilon}, \gamma_{0}=\frac{i \omega_{0}}{\Omega}, \\
\alpha_{\perp}=\frac{n_{i}}{n_{e}} \gamma_{0}, \alpha_{\|}=\alpha_{\perp}(2 N+1) .
\end{gathered}
$$

The left-hand side in Eq. (25) is nothing but the dispersion equation for electromagnetic waves in layered conductors without the corrections due to the magnetoimpurity bound states. The quantity $V\left(\alpha_{\|}, \alpha_{\perp}\right)$ depends on the impurities concentration $n_{i} / n_{\varepsilon}$ and vanishes when $n_{i}=0$.
Consider first the case of waves propagating along the magnetic field. These waves have zero component along the planes, $q=0$ and, therefore, the structure form-factor $S(q, k, \omega)(22)$ becomes

$$
S(q, k, \omega) \approx \frac{i \omega_{*} \tilde{\omega} n_{0}}{1-\cos (k a)},
$$

where $\tilde{\omega}=\omega / \omega_{p}, \omega_{p}=4 \pi n_{e} e^{2} / m a$ is the plasma frequency; $n_{0}=\sqrt{\varepsilon_{0}}$ is the refraction index of the matter between the layers.

The form-factor (29) is small elsewhere except for $k a<<1$ because of the smallness of the parameter $\omega_{*} \tilde{\omega} n_{0}<<1$. Putting $n_{i}=0$ and substituting Eq. (29) into (25) we found

$$
\begin{gathered}
1+(g-i X)^{2}-i(g-i X) 2 A X-A^{2} X^{2}=0, \\
g=\frac{v}{\Omega}, X=\frac{\omega}{\Omega}, A=\left(\frac{\omega_{*}}{2}\right)^{2} \sin ^{-2}(k a / 2) .
\end{gathered}
$$

The dispersion equation (30) has exact solutions (1) and (2), that is typical for helicons. The resonances at $\omega \approx \omega_{0}$ brought into the system, owing to the magnetoimpurity bound states, should modify the helicons dispersion near the intersection of the dispersion curves $\omega(k)$ (1) and $\omega=\omega_{0}$. Qualitatively, the impact of this «crossing event» on the wave spectrum is well known [18]: a twofold degeneracy is lifted up, causing the appearance of two new branches in the dispersion law of helicons, one above and another below $\omega_{0}$. To consider this phenomenon in more detail, we should include the $V$-term (26) in the right-hand side of dispersion equation (30).

Taking into account the smallness of the impurity concentration and the condition $\omega_{0} / \Omega<<1$, we have

$$
\begin{aligned}
1+ & (g-i X)^{2}-i(g-i X) 2 A X-A^{2} X^{2} \\
& =\frac{2 \alpha_{\perp}}{X-X_{0}+i g_{0}} A X(A X-B) .
\end{aligned}
$$

Here

$$
B=2 N+1, X_{0}=\frac{\omega_{0}}{\Omega}, g_{0}=\frac{v_{0}}{\Omega},
$$

and $v_{0}$ is the broadening of the bound state $\omega_{0}$.

We see that the right-hand side of Eq. (32) (which is due to the magnetoimpurity bound states) enhances the degree of algebraic Eq. (32) compared with Eq. (30) and bring forth an additional root $X=X\left(k, A, g, g_{0}\right)$, i.e., a new branch in the wave dispersion equation. 


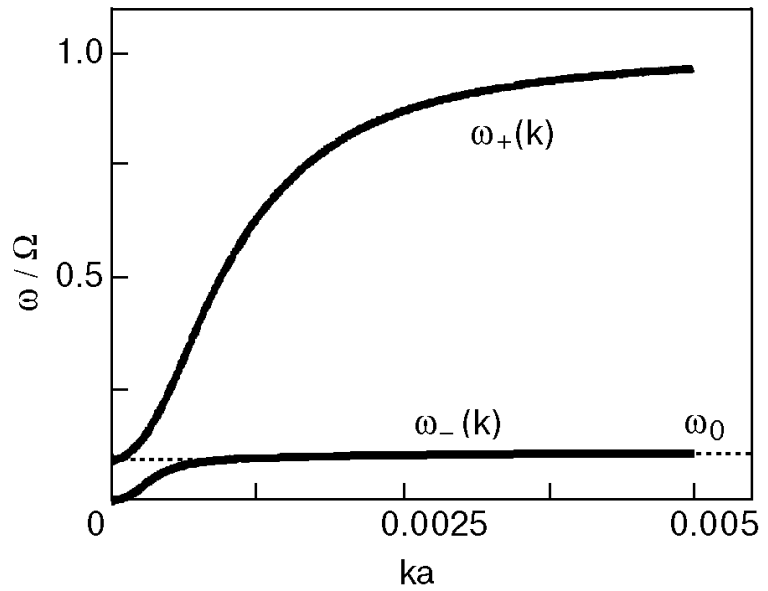

Fig. 1. The high-frequency helicon-like mode $\omega_{+}(k)$ and the low-frequency magnetoimpurity mode $\omega_{-}(k)$ calculated numerically from the dispersion equation (32) for $\omega_{*}=0.01, X_{0}=0.1$, $\alpha_{\perp}=0.1, \alpha_{\|}=10, g=g_{0}=0$.

The results of numerical calculations for the dispersion curves given by Eq. (32) are shown in Fig. 1.

The most dramatic changes are seen to take place near the crossing point of two branches: the helicon dispersion branch $\omega(k)$, given by Eq. (1), and the magnetoimpurity (bound state energy) branch $\omega=\omega_{0}$. They are shown in more detail in Fig. 2 . We see that two new modes appear in consequence of this crossing : the low-frequency mode $\omega_{-}(k)$ and the high-frequency mode $\omega_{+}(k)$. The low-frequency mode dispersion for $k a<<1, g=0, g_{0}=0$ is given by

$$
\omega_{-}(k) \approx \Omega^{2}(k a)^{2} \frac{2 \alpha_{\perp} B}{\omega_{*}^{2} \omega_{0}}\left\{\left[1+\left(\frac{\omega_{0}}{\Omega \alpha_{\perp} B}\right)^{2}\right]^{1 / 2}-1\right\} .
$$

For $k a \simeq 1, g=0, g_{0}=0$ it behaves as

$$
\omega_{-}(k) \approx \omega_{0}-\frac{\Omega \omega_{*}^{2} \alpha_{\perp} B}{\sin (k a / 2)} .
$$

In the same fashion one can easily obtain explicit relations for the high-frequency mode $\omega_{+}(k)$. In the case of zero broadening $\left(g=g_{0}=0\right)$, we have

$$
\omega_{+}(k) \approx \omega_{0}-2 \alpha_{\perp} \Omega\left[1-\frac{B \Omega}{\omega_{0} \omega_{*}^{2}}(k a)^{2}\right] \quad \text { for } k a<<1
$$

and

$$
\omega_{+}(k) \approx \Omega\left[1+\frac{\alpha_{\perp} B \omega_{*}^{2}}{4 \sin ^{2}(k a / 2)}\right] \quad \text { for } k a \simeq 1 .
$$

From the numerical calculations shown in Fig. 2, it follows that there is no gap between the branches $\omega_{+}(k)$ and $\omega_{-}(k)$ for $v=0$, since $\omega_{+}(0)<\omega_{0}$. This gap appears for nonzero Landau levels broadening as one can see from Fig. 2,b, where calculations are made for $v=0.01$ and it is found that $\omega_{+}(0)>\omega_{0}$, which is the upper limit of the low-frequency mode $\omega(k)$.

In case of nonzero $g$ and $g_{0}$ both modes, $\omega_{+}(k)$ and $\omega_{-}(k)$, are damped. In fact, only weakly damped modes can propagate in a layered conductor, which implies parameters $g$ and $g_{0}$ should be much less than unity. Writing the wave frequency in the complex form $\omega=\operatorname{Re} \omega-i \operatorname{Im} \omega$ (i.e., taking into account its damping) we found from Eq. (30) in the linear on $g$ and $g_{0}$ approximation,
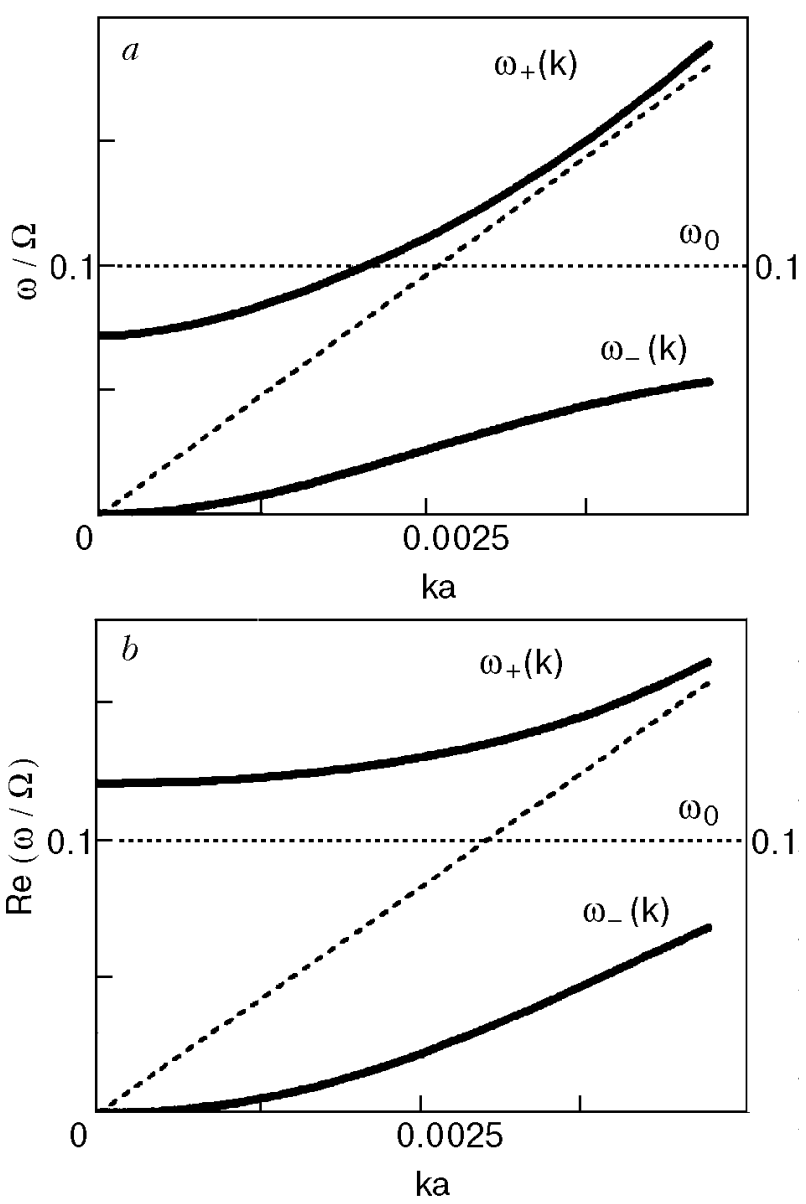

Fig. 2. The $\omega_{+}(k)$ and $\omega_{-}(k)$ modes (as in Fig. 1) shown in more detail near the crossing point of helicons (dashed line) with the magnetoimpurity frequency $\omega_{0}$ (dots). In difference to Fig. 1 parameters $\alpha_{\perp}$ and $\alpha_{\|}$are taken to be $\alpha_{\perp}=0.01$ and $\alpha_{\|}=100$ for more descriptiveness of the picture. The absence of the gap between modes $\omega_{+}(k)$ and $\omega_{-}(k)$ is seen in Fig. 2, $a$ (where $g=0$ ) since $\omega_{+}(0)<\omega_{0}$. For slightly damped Landau levels $g \neq 0$ the above gap may appear, as is the case in Fig. 2,b, where $\operatorname{Re} \omega_{+}(0)>\omega_{0}$ and $g=0.01$. 


$$
\begin{gathered}
\operatorname{Im} \omega_{ \pm}(k)=\frac{\Omega}{2}\left\{\frac{\omega_{ \pm}(k)}{\Omega}\left[b-d \frac{\omega_{ \pm}(k)}{\Omega}\right]+g_{0}\right\} \times \\
\quad \times\left\{a+2 \frac{\omega_{ \pm}(k)}{\Omega}\left[c-3 e \frac{\omega_{ \pm}(k)}{\Omega}\right]\right\}^{-1},
\end{gathered}
$$

where

$$
\begin{gathered}
a=1+2 \alpha_{\perp} A B, b=2 g X_{0}(1+A), \\
c=X_{0}(1+A)^{2}-2 \alpha_{\perp} A^{2}, \\
d=g_{0}\left(1-2 A-A^{2}\right)+2 g(1+A), e=(1+A)^{2} .
\end{gathered}
$$

The numerical calculations for damping of the low-frequency, $\omega_{-}(k)$ and high-frequency, $\omega_{+}(k)$, modes are shown in Fig. 3.

\section{Summary and conclusions}

We have shown above that magnetoimpurity states which appear in layered conductors in a quantizing external magnetic field are favorable for the propagation of the electromagnetic waves across the layers. These new modes come into being near the frequencies of the electron resonant transition between the magnetoimpurity level $\omega_{0}$ and the Landau levels. Physically, the magnetoimpurity modes do exist because of the electron localization at the impurity atoms which is favored by the external magnetic field and the two-dimensional nature of the electron dynamics within the layers. This localization made conductors closer to dielectrics which are known to be transparent for the electromagnetic waves. The magnetoimpurity waves in conventional $3 D$ metals have been predicted by Kaner and Ermolaev [19,20] and then considered theoretically in the so-called noncompensated me-

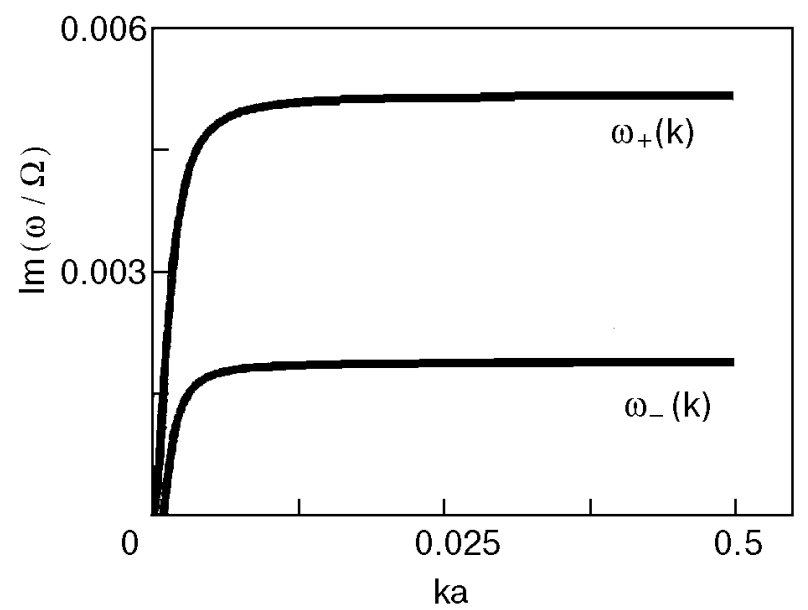

Fig. 3. The damping of the $\omega_{+}(k)$ and $\omega_{-}(k)$ modes shown in Fig. 1 calculated for $g=0.01$ and $g_{0}=0.01$.

tals in which the electric charges are carried both by electrons and holes [18]. The quasi-two-dimensional character of the problem considered in this paper makes the whole phenomenon more pronounced, because of more strong van Hove singularities in the density of electronic states $g(\omega)$ in $2 D$ systems compared to $3 D$ ones. The latter facilitates the creation of a bound state $\omega_{0}$ (i.e., the eigenvalue of the Lifshitz equation, which we took for granted above) and, in particular, enhances the singularity in the frequency dependences of the $\delta \sigma_{x x}(\omega)$ in Eqs. (14)-(15) compared to the $3 D$ case. Recall, that quantities $\delta \sigma_{x x}(\omega)$ and $\delta \sigma_{x y}(\omega)$ have square-root singularities in the $3 D$ case that arise due to the appropriate singularities of the $g(\omega)$ at the Landau levels and do not depend on the details of the attractive potential of the impurities.

The spiral magnetoimpurity waves, to the best of our knowledge, have not yet been observed, although the existence of the magnetoimpurity bound states was surely established in some doped $\mathrm{Bi}$ compounds [21-23] and semiconductors [24].

In layered conductors, the conditions for bonding of electrons by the attractive impurities in an external magnetic field are more favorable than in $3 D$ metals, which enhances the chance of finding magnetoimpurity waves in such systems. Currently, a good deal of layered conductors (natural and artificially fabricated) are under study and attraction of researchers to the problem of magnetoimpurity waves in them is one of the purposes of this paper. Unfortunately, the most popular new layered systems, namely $\mathrm{Bi}-$ and Tl-based high- $T_{c}$ cuprates, should be, to all appearances, excluded from the list of candidates for observation of the magnetoimpurity modes in them because of the large damping $v \simeq \omega$ found in these materials above $T_{c}[25]$.

1. E. A. Kaner and V. G. Skobov, Adv. Phys. 17, 605 (1968).

2. A. A. Abrikosov, Fundamentals of the Theory of Metals, North-Holland, Amsterdam (1988).

3. V. G. Skobov, Zh. Exper. Teor. Fiz. 37, 1467 (1959) [Sov. Phys. JETP 10, 1039 (1960)].

4. Yu. A. Bychkov, Zh. Exper. Teor. Fiz. 39, 689 (1960) [Sov. Phys. JETP 12, 483 (1961)].

5. N. V. Gleizer and A. M. Ermolaev, Fiz. Nizk. Temp. 23, 73 (1997) [Low Temp. Phys. 23, 55 (1997)].

6. F. G. Bass, A. A. Bulgakov, and A. P. Tetervov, High-frequency Properties of Semiconducting Superlattices, Nauka, Moskow (1989) [in Russian].

7. V. M. Gohfeld, M. I. Kaganov, and V. G. Peschansky, Fiz. Nizk. Temp. 12, 1173 (1986) [Sov. J. Low. Temp. Phys. 12, 661 (1986)].

8. V. G. Peschansky, H. Kheir Bek, and S. N. Savel'eva, Fiz. Nizk. Temp. 18, 1012 (1992) [Low Temp. Phys. 18, 711 (1997)].

9. A. Tselis and J. J. Quinn, Phys. Rev. B29, 3318 (1984). 
10. K. I. Golden and G. Kalman, Phys. Rev. B52, 14719 (1995).

11. L. Wendler and M. I. Kaganov, Phys. Status Solidi B142, K63 (1987).

12. B. N. Narahari Achar, Phys. Rev. B37, 10423 (1988).

13. I. D. Vagner and D. Bergman, Phys. Rev. B35, 9856 (1987).

14. V. M. Gvozdikov, Physica C224, 293 (1994).

15. V. M. Gvozdikov and R. Vega-Monroy, Vestnik Kharkovs kogo Universiteta 118, 4 (1998).

16. L. D. Landau and E.M. Lifshitz, Quantum Mechanics, Nauka, Moskow (1989).

17. T. Ando, A.B. Fowler, and F. Stern, Rev. Mod. Phys. 54, 437 (1982)
18. N. V. Gleizer, A. M. Ermolaev, and G. I. Rashba, Fiz. Nizk. Temp. 20, 1169 (1994) [Low Temp. Phys. 20, 919 (1994)].

19. E. A. Kaner and A. M. Ermolaev, Pis'ma Zh. Exp. Teor. Fiz. 44, 3915 (1986)

20. E. A. Kaner and A. M. Ermolaev, Zh. Exp. Teor. Fiz. 92, 2245 (1987) [Sov. Phys. JETP 65, 1266 (1987)].

21. A. M. Ermolaev and M. I. Kaganov, Pis'ma Zh. Exp. Teor. Fiz. 6, 984 (1967) [JETP Lett. 6, 315 (1967)].

22. A. M. Ermolaev, Zh. Exp. Teor. Fiz. 54, 1259 (1968) [Sov. Phys. JETP 27, 673 (1968)].

23. N. B. Brandt and L. G. Lyubutina, Zh. Exp. Teor. Fiz. 52, 686 (1967) [Sov. Phys. JETP 25, 450 (1967)].

24. S. P. Andreev, Usp. Fiz. Nauk. 143, 213 (1984) [Sov. Phys. Usp. 27, 431 (1984)].

25. J. Ruvalds, Supercond. Sci. Technol. 9, 905 (1996). 